\section{Aflibercept as a Second Line Therapy for Neovascular Age Related Macular Degeneration in Israel (ASLI) study}

${ }^{1}$ Department of

Ophthalmology Hadassah, Hebrew University Medical Center, Jerusalem, Israel

${ }^{2}$ Meir Medical Center, Kfar Sava, affiliated to the Sackler School of Medicine, Tel Aviv University, Israel

${ }^{3}$ Carmel Medical Center, Haifa, Israel

${ }^{4}$ Kaplan Medical Center, Rehovot, Israel

${ }^{5}$ Rabin Medical Center, Sackler Faculty of Medicine, Tel Aviv University, Israel

${ }^{6}$ Soroka University Medical Center, Beer Sheva, Israel

${ }^{7}$ Rambam Medical Center, Haifa, Israel

${ }^{8}$ Sheba Medical Center, Tel Hashomer, Israel

${ }^{9}$ Tel Aviv Medical Center, Sackler Faculty of Medicine, Tel Aviv University, Israel

Correspondence:

I Chowers, Department of Ophthalmology Hadassah, Hebrew University Medical Center, POB 12000, Jerusalem 91120, Israel

Tel: +972 53 3312450;

Fax: +97226777228

E-mail: chowers@

hadassah.org.il

${ }^{10}$ These authors contributed equally to this work.

Received: 26 May 2016 Accepted in revised form:

5 December 2016

Published online:

17 February 2017

\section{Abstract}

Purpose The purpose of this study is to evaluate an early switch to aflibecept in eyes with neovascular age-related macular degeneration (nvAMD) showing partial or lack of response for initial therapy with bevacizumab.

Methods The Aflibercept as a Second Line Therapy for Neovascular Age Related Macular Degeneration in Israel (ASLI) was a prospective, multicenter, single-arm clinical trial. Eyes with nvAMD having incomplete response to 3-9 prior bevacizumab injections were recruited. Three monthly intravitreal aflibercept (2 $\mathrm{mg}$ ) injections were administered, followed by two bi-monthly injections and a final examination at week 28 . An optional injection was allowed at week 20.

Results Forty-seven eyes of 46 patients (mean \pm SD age $76 \pm 8$ years) were recruited. The mean number of prior bevacizumab injections was $5.5 \pm 2.9$. The mean visual acuity improved from $60.3 \pm 10$ ETDRS letters at baseline to $63.1 \pm 15$ letters at week 28 $(P=0.02$, paired $t$-test). The central subfield thickness (CST) reduced from $409 \pm 127$ micron at baseline to $330 \pm \mathbf{1 1 0}$ microns at week 4 ( $P=0.0002$; paired $t$-test), and $277 \pm 70$ microns at week $28(P=0.00002$; paired $t$-test $)$. Twenty-two eyes had three to five prior bevacizumab injections (mean $5.1 \pm 0.7$ ), and 25 eyes had six to nine prior injections $(7.32 \pm 1.2)$. Both groups had reduced CST from baseline to week $28(P=0.0004$ and $P=0.0007$; paired $t$-test, respectively). Thirtyfive $(75 \%)$ eyes required the optional additional aflibercept injection at week 20 . Conclusions The ASLI study demonstrated improved BCVA and reduced CST following an early switch to aflibercept therapy in eyes
L Tiosano1, O Segal ${ }^{2,10}$, N Mathalone ${ }^{3,10}$ A Pollack ${ }^{4}$, R Ehrlich ${ }^{5}$, I Klemperer ${ }^{6}$, Y Barak ${ }^{7}$, I Moroz ${ }^{8}$, I Chowers ${ }^{1}$ and M Goldstein ${ }^{9}$

with prior incomplete response to initial therapy with three to nine bevacizumab injections.

Eye (2017) 31, 890-898; doi:10.1038/eye.2017.7; published online 17 February 2017

Introduction

Anti-VEGF compounds, administered intravitreally, markedly improved the short- and intermediate-term outcome of neovascular agerelated macular degeneration (nvAMD). On the basis of several prospective multicenter clinical trials, it was concluded that non-inferior visual outcome is obtained when either ranibizumab or bevacizumab is applied for treating nvAMD. ${ }^{1-5}$ The VEGF-Trap Eye Investigation of Efficacy and Safety in Wet AMD (VIEW) 1 and VIEW 2 trials also described similar visual outcome following either ranibizumab or aflibercept treatment for nvAMD. ${ }^{6-8}$

Aflibercept is a recombinant fusion protein consisting of the key human VEGF receptor extracellular domains from receptors 1 and 2 (VEGFR1 and VEGFR2) fused to the Fc domain of human IgG1. ${ }^{9}$ Aflibercept is different from bevacizumab and ranibizumab by having higher binding affinity for VEGF-A by binding to placental growth factors 1 and 2 (PLGF1 and PLGF2) and VEGF-B, and by having a longer half-life in the vitreous (compared to ranibizumab). ${ }^{7,10}$ While, overall, bevacizumab, ranibizumab, and aflibercept obtain similar treatment outcome in nvAMD, differences among these compounds may affect treatment outcome in the subgroup of patients.

The VIEW 1 and VIEW 2 studies demonstrated that aflibercept administered every 2 months following three initial monthly loading doses was non-inferior to monthly ranibizumab 
in maintaining vision in patients with treatment-naive nvAMD over 1 year. ${ }^{6}$ Yet, a recent study reported that a more frequent injection schedule may be required in some of the cases. ${ }^{11}$

Despite the similar overall efficacy, when unsatisfactory response is observed following treatment with a single anti-VEGF compound, a switch to one of the other available anti-VEGF drugs is often performed. This strategy is based on several reports, suggesting anatomic and visual outcome benefits following switching between anti-VEGF therapies in cases of unfavorable response to the first-line treatment. ${ }^{8,12-33}$

According to the program of the Israeli Ministry of Health, off-label bevacizumab serves as the first-line therapy for nvAMD in the majority of nvAMD cases. Guidelines for the preferred timing and benefit of switch to a second-line therapy for nvAMD were not developed at the inception of this study. In many cases, such secondline treatment is initiated only after multiple previous injections of the first-line compound. By that time a substantial visual loss and structural macular damage have already occurred. Conceivably, an early switch, when irreversible damage to foveal tissue is still limited, may yield improved outcome.

To further assess the efficacy of aflibercept as an early second-line therapy in nvAMD, we have conducted a multicenter prospective clinical trial in nvAMD patients with lack or incomplete response to prior first-line bevacizumab treatment.

\section{Materials and methods}

\section{Study design}

The Aflibercept as Second Line treatment in Israel (ASLI) study was a prospective, multicenter, open label, singlearm clinical trial that was initiated by the Israeli Retina Society. Forty-six patients (47 eyes) were enrolled during 12 months from April 2014 through April 2015 at eight clinical centers around Israel. All patients were followed for 28 weeks. The study was approved by the institutional review board at each participating clinical center, and all patients provided written informed consent. The ASLI study was registered with ClinicalTrials.gov (identifier no. NCT01918878).

\section{Participants}

Inclusion criteria for the study eye included incomplete or no response to first-line intravitreal injections of bevacizumab for nvAMD. First-line treatment was provided in the setting of a clinic prior to recruitment to the ASLI trial. Incomplete or no response was defined based on OCT criteria that included the presence of intra- or subretinal fluid (IRF, SRF), and/or pigment epithelium detachment (PED) with maximal central point thickness of 300 microns or larger (retinal thickness including SRF, IRF, and PED) according to the Heidelberg OCT. We included patients who received between three and nine bevacizumab injections within the first year from diagnosis of new onset choroidal neovascularization (CNV) secondary to AMD. The intervals between each of the last three injections had to be no longer than 6 weeks. This interval was chosen as it reflected real-life clinic setting treatment interval, and since it was considered acceptable at the time. ${ }^{34-38}$ Best-corrected visual acuity (BCVA) in the study eye was between 30 and 80 letters of Early Treatment Diabetic Retinopathy Study (ETDRS) visual acuity chart (Snellen equivalent of $\sim 20 / 40$ to $20 / 360$ ). Inclusion imaging criteria were based on optical coherence tomography (OCT) and fluorescein angiogram (FA) performed at the screening visit. FA inclusion criteria included total area of the lesion (including leakage, blood, and scar/atrophy) smaller than five disc areas, of which at least 50\% must be active CNV. Exclusion criteria included presence of any pathology, other than nvAMD, that could be associated with the development of $\mathrm{CNV}$, more than $50 \%$ of the total lesion size consisting of subretinal hemorrhage, presence of retinal angiomatous proliferation, or RPE tears. Active or suspected ocular or periocular infection or inflammation, clinically significant epiretinal membrane, vitreomacular traction, macular hole, or vitreous hemorrhage were other exclusion criteria. Eyes with uncontrolled intraocular pressure (IOP) or any intraocular surgery or thermal laser within 3 months of trial entry were also excluded.

\section{Study protocol}

BCVA and imaging (OCT and FA) were performed following a standardized protocol. OCT images from the screening visit were reviewed by the site's PI. FA images were analyzed by a centralized reading center that reviewed all FAs to determine eligibility (MG). To approve eligibility for the study, the FA had to be deemed eligible by the reading center, and the clinical details and OCT images had to be reviewed by additional two PIs from other participating centers. A unanimous consensus was required to confirm participation of the patients in the study.

Loading dosage composed of three monthly intravitreal aflibercept ( $2 \mathrm{mg}$; day 0 , week 4 , and week 8 ) was followed by further injections of aflibercept at weeks 16 and 24. In cases where IRF and/or SRF were detected at week 16, an additional injection was provided at week 20 per the investigator's discretion. Thus, a total of five to six aflibercept injections were administered during the study period. 
At each visit, a full ophthalmic examination, including protocol BCVA and IOP assessment with Goldman applanation tonometry, adverse event monitoring, and SD-OCT / HD-OCT (Heidelberg Spectralis; Heidelberg Engineering, Heidelberg, Germany, or Ziess cirrus, Carl Zeiss Meditec, Dublin, CA, USA), measurement of both eyes was conducted with appropriate correction factors of adding 10 microns to the thickness for measurements that were based on Cirrus HD-OCT to compare thickness to the Spectralis SD-OCT. ${ }^{39}$ FA was performed at the baseline at the last visit.

\section{Statistical analysis}

Statistical analysis was performed using SPSS (version 21.0; SPSS Inc., Chicago, IL, USA). Study sample was calculated using Hochberg's procedure to control the type I error rate for the primary end point at 5\% (two-sided), and provide $80 \%$ probability that the change in the central subfield thickness (CST) will be significant. Measures of BCVA, CST, and maximal central thickness were summarized using means, SDs, median, and range. Since the data were normally distributed, comparisons with and between groups for BCVA and CST parameters were performed using two-sided $t$-tests. Where appropriate, sensitivity analysis using nonparametric Wilcoxon-signed rank tests was also performed. A $P$-value of less than 0.05 was considered significant.

\section{Results}

\section{Baseline patient characteristics and patient retention}

Forty-seven eyes of 46 patients were enrolled to the study in eight academic medical centers around Israel. The mean \pm SD age at baseline was $76 \pm 8$ years, and $28(61 \%)$ patients were female. Twenty-two eyes had three to five prior bevacizumab injections (mean \pm SD $5.1 \pm 0.7$ ), and 25 eyes had six to nine prior injections (mean \pm SD $7.32 \pm 1.2$ ). Baseline characteristics are presented in Table 1.

One patient was withdrawn from the study at week 16 because of unsatisfactory response according to the discretion of the treating PI. This patient manifested exacerbation of PED and adjacent subretinal fluid despite 3 monthly injections of afllebercept. One patient did not receive the treatment at week 16 because of an active herpes simplex keratitis in the study eye. When the infection resolved, the remaining treatments were provided as scheduled. Three ocular adverse events were observed, one eye had retinal detachment during the follow-up, and two eyes had transient elevated IOP. One patient experienced bowel obstruction, which was treated medically without the need for surgery.
Table 1 Baseline characterization of patients enrolled within the ASLI study $(n=47)$

\begin{tabular}{|c|c|}
\hline Age (years) mean $\pm \mathrm{SD}$ & $76 \pm 8$ \\
\hline Female/male $(\%)$ & $61 / 39$ \\
\hline \multicolumn{2}{|c|}{ Previous bevacizumab treatment, number (\%) } \\
\hline 3 & $15(32 \%)$ \\
\hline 4 & $4(8 \%)$ \\
\hline 5 & $3(6 \%)$ \\
\hline 6 & $9(19 \%)$ \\
\hline 7 & $7(15 \%)$ \\
\hline 8 & $1(2 \%)$ \\
\hline 9 & $8(17 \%)$ \\
\hline \multicolumn{2}{|c|}{ Vitreoretinal interface, number (\%) } \\
\hline Attached vitreous & $18(38 \%)$ \\
\hline PVD & $15(32 \%)$ \\
\hline ERM & $1(2 \%)$ \\
\hline VMT & $1(2 \%)$ \\
\hline \multicolumn{2}{|l|}{ Drusen type, number (\%) } \\
\hline Typical AMD & $36(77 \%)$ \\
\hline Cuticular & $3(6 \%)$ \\
\hline Subretinal drosenoid & $3(6 \%)$ \\
\hline \multicolumn{2}{|l|}{ Retinal fluid, number (\%) } \\
\hline SRF & $33(70 \%)$ \\
\hline IRF & $18(38 \%)$ \\
\hline PED & $21(45 \%)$ \\
\hline
\end{tabular}

Abbreviations: AMD, age-related macular degeneration; ERM, epiretinal membrane; IRF, intraretinal fluid; PED, pigment epithelium detachment; PVD, posterior vitreous detachment; SRF, subretinal fluid; VMT, vitreomacular traction.

\section{Anatomic outcome}

The CST reduced during the study from a mean \pm SD of $409 \pm 127$ microns at baseline to $330 \pm 100$ microns $(P=0.0002)$ at week 4 and $277 \pm 70$ microns $(P=0.00002$; paired $t$-test) (Figure 1a) at week 28. Following the first bi-monthly interval between the week 8 and week 16 visits, there was an increase in CST from $324 \pm 112$ to $358 \pm 125$ microns $(P=0.003$; paired $t$-test). Overall, $35(75 \%)$ eyes received the optional injection at week 20. The CST remained stable in the 12 eyes that received only the two bi-monthly injections at weeks 16 and 24 (Figure 1b).

Subgroup analysis for 22 eyes that had three to five prior bevacizumab injections, and 25 eyes with prior six to nine injections demonstrated reduction in CST in both groups from mean \pm SD of $407 \pm 145$ and $405 \pm 107$ microns at baseline to $280 \pm 84$ and $272 \pm 51$ microns at week $28(P=0.0004$ and $P=0.0007$, respectively; paired $t$-test; Figure 1c). Both groups demonstrated similar visual outcome during the follow-up (Figure 1c).

Thirty-three $(70 \%)$ eyes had SRF at presentation, while only $12(25 \%)$ eyes had SRF at week $28(P=0.01)$. Only four ( $8 \%$ ) eyes had IRF at week 28 compared with 18 eyes $(38 \%)$ at baseline $(P=0.01)$. PED was present in 18 

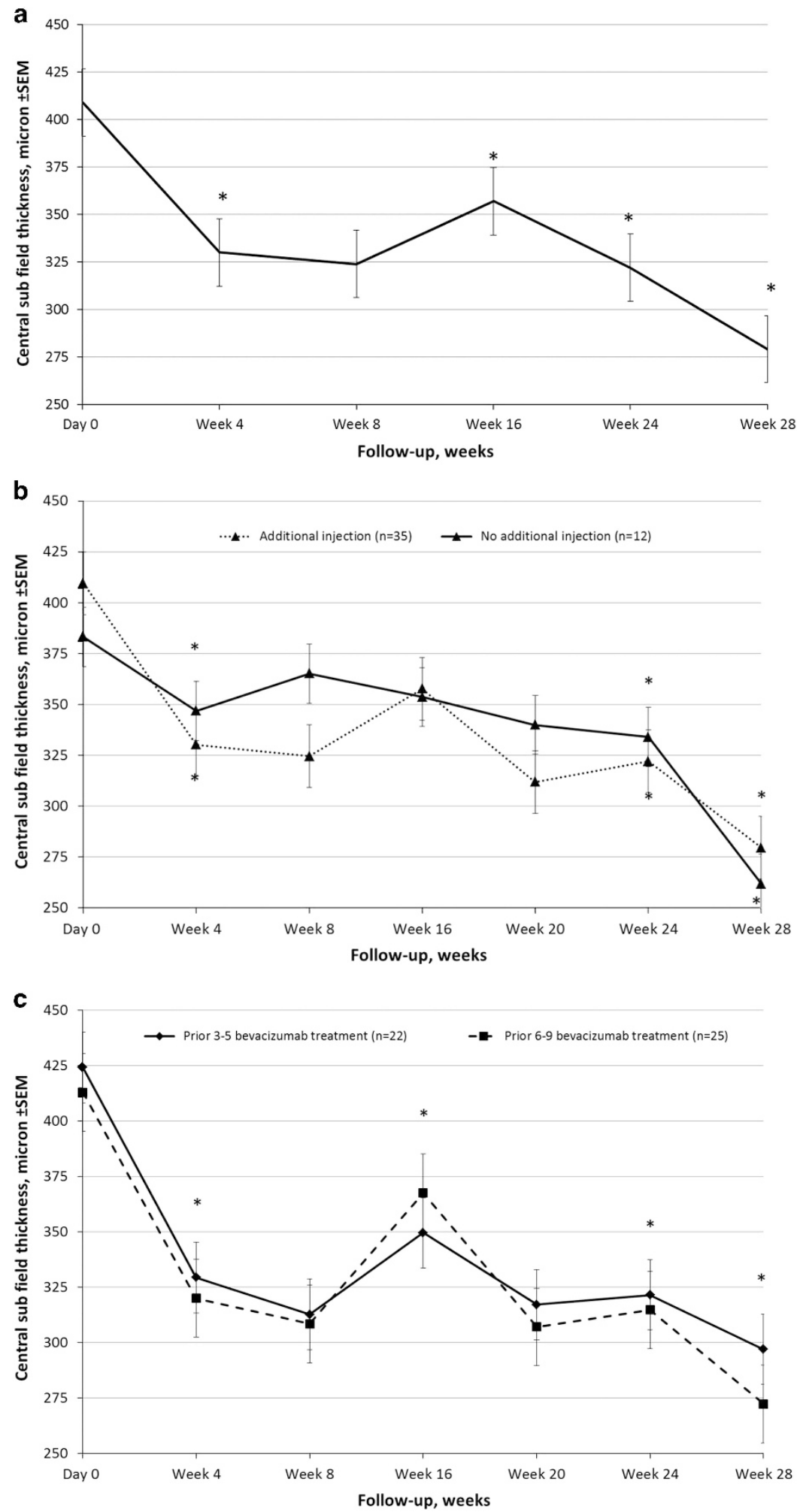

Figure 1 The mean change in CST in microns according to OCT during 28 weeks of follow-up $(n=47 ; \mathrm{a})$. (b-c) A subgroup analysis of CST changes during the study. (b) CST changes in eyes that received additional injection at week $20(n=35)$ and eyes without additional injection $(n=12)$. There were similar CST changes in both groups. (c) CST changes along the study according to the number of bevacizumab injections prior to enrollment to the study. There were similar CST changes in both groups. ${ }^{*} P<0.05$ for the comparison of baseline $v$ f follow-up examination in the same group. Error bars represent SEM. 
eyes (38\%) at week 28 compared with 21 eyes (45\%) at baseline $(P=0.8)$.

\section{Visual acuity outcome}

BCVA score improved during the study from mean \pm SD at baseline of $60.3 \pm 10$ to $63.1 \pm 15$ letters at the end of the follow-up ( $P=0.02$; paired $t$-test; Figure 2a). At week 28, $21(45 \%)$ eyes improved by five letters or more and three (6\%) eyes improved by 15 letters or more. Thirteen $(28 \%)$ eyes lost one or more letter. Six (12\%) eyes lost less than five ETDRS letters, three (6\%) eyes lost 5-15 letters, and four $(8 \%)$ eyes lost more than 15 letters during the study. Subgroup analysis identified no difference in visual outcome between the 35 eyes that had additional injection at week 20 and the remaining 12 study eyes $(P=0.73$; Figures $2 b$ and 3 ). Subgroup analysis for 22 eyes with three to five prior bevacizumab injections demonstrated significant BCVA improvement from $60.4 \pm 11.2$ at baseline to $62.8 \pm 18.7$ ETDRS letters at week $28(P=0.04$; paired $t$-test). A trend of BVCA improvement was detected for the 25 eyes with prior six to nine injections from $62.9 \pm 8.5$ ETDRS letters at baseline to $65.2 \pm 11.6$ ETDRS letters at week $28(P=0.23$; paired $t$-test; Figure 2c).

\section{Discussion}

In this prospective multicentre study, intravitreal aflibercept therapy as an early second-line therapy following bevacizumab failure yielded anatomical and functional improvement. The mean macular thickness reduced by over 100 micron and qualitative OCT parameters improved. The mean visual acuity increased by one ETDRS line or more in $45 \%$ of patients.

Multiple authors described outcomes after aflibercept therapy in cases of incomplete response to prior therapy with bevacizumab or ranibizumab in nvAMD. ${ }^{8,14-29,31-33}$ Recently, Lazzeri et al ${ }^{40}$ summarized these studies. Of the 21 studies identified, 16 were retrospective and 5 were prospective. ${ }^{14,17,21,26,32}$ Unlike the ASLI study, the majority of patients in the previous prospective studies were treated with first-line ranibizumab and because of partial response were switched to aflibercept. These prospective studies included all together 157 eyes with a follow-up period of 6-12 months. The mean number of anti-VEGF injections prior to the switch to aflibercept was 30 , as opposed to our study in which the number was significantly lower (between three and nine). There were large variations in the number of prior injections within studies. Overall, the prospective studies reported a mean reduction of $\sim 50$ microns in macular thickness at 6 months and a mean improvement of four letters in the three studies who reported ETDRS visual acuity. ${ }^{40}$
Sarao et $a l^{30}$ recently reported another prospective study that was not included in the meta-analysis of Lazzeri et al. ${ }^{40}$ In this relatively large study $(n=92)$, similar to our finding, an improvement of 1.8 letters in visual acuity and reduction in CST of over 100 microns was reported. Eyes were treated with aflibercept following an initial PRN protocol with ranibizumab injections and monthly monitoring and an average of 3.5 injections during 12 months of follow-up. ${ }^{30}$ Prior to switching to aflibercept, these eyes received an average of $\sim 15$ injections during 18 months of therapy.

Overall, results of these previous prospective studies are in accordance with our findings. The current trial provided additional important insights into second-line aflibercept treatment. Unlike previous prospective studies, the ASLI trial focused on systematic assessment of early conversion to second-line aflibercept and of the required treatment interval period in such eyes. Such early conversion may theoretically prevent the atrophic macular changes and scar formation, which are often observed in eyes with persistent CNV activity. Despite this theoretical advantage, the magnitude of visual gain in our study is in the same order of previous studied where second-line therapy was initiated after longer periods of first-line therapy. Furthermore, there was no difference in outcome between eyes with less ${ }^{3-5}$ or more ${ }^{6-9}$ prior bevacizumab injections within the ASLI trial. Thus, conceivably, the benefit of early rather than delayed conversion to aflibercept is not reflected in the absolute visual gain following the conversion. Yet, it is likely that early conversion to successful second-line therapy may result in better outcome by avoiding the visual loss, which occurs during the time period of treatment with lesseffective therapy for the particular eye.

According to its label for treatment of nvAMD, three monthly intravitreal injections of aflibercept are initially provided followed by bi-monthly injections. We have adopted this treatment schedule but also allowed for a rescue treatment at week 20 , following the initial bimonthly interval in cases where IRF and/or SRF were detected at week 16. Interestingly, we have found that average macular thickness increased after the first bimonthly injection and that more than half of the patients required monthly aflibercept injection during the first 6 months of second-line therapy. Accordingly, it was recently suggested that bi-monthly aflibercept may not be sufficient in some of the nvAMD patients who receive this compound as a first-line therapy. ${ }^{11}$

The main caveats of our study is the lack of a control group, the small number of patients and relatively short follow-up period. It is not possible to assure that similar anatomical and visual gains will not be detected following longer treatment with first-line therapy, or following strict q4-week treatment schedule with 
a
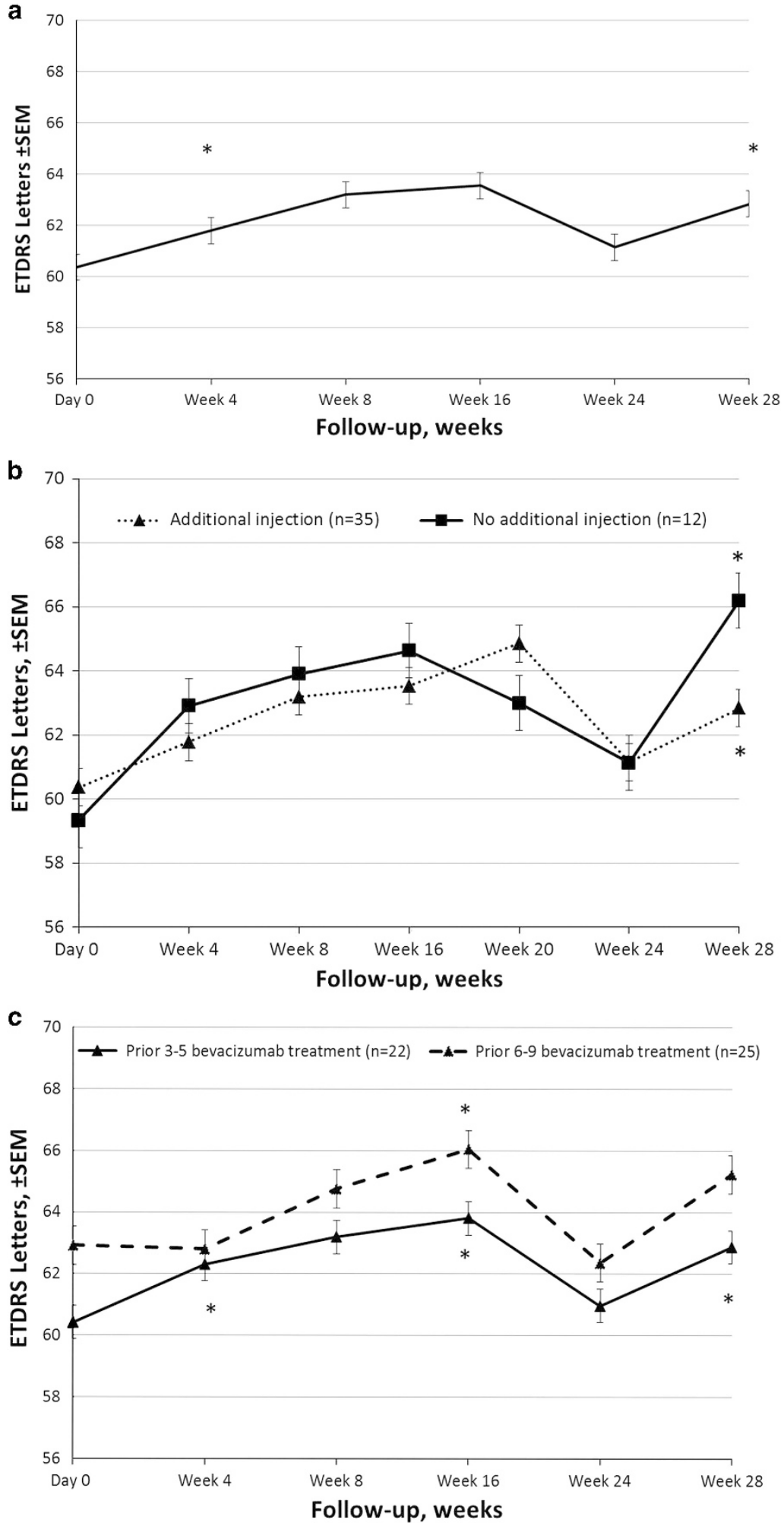

Figure 2 The mean change in BCVA measured by ETDRS letters $(n=47)$ during the study period (a). (b-c) A subgroup analysis of the mean change in BCVA during the study. (b) BCVA changes in eyes that received additional injection at week $20(n=35)$, and in eyes without additional injection $(n=12)$. (c) BCVA changes along the study according to the number of bevacizumab injections prior to enrollment to the study. There were similar BCVA changes in both groups. ${ }^{*} P<0.05$ for the comparison of current vs follow-up examination. Error bars represent SEM. 

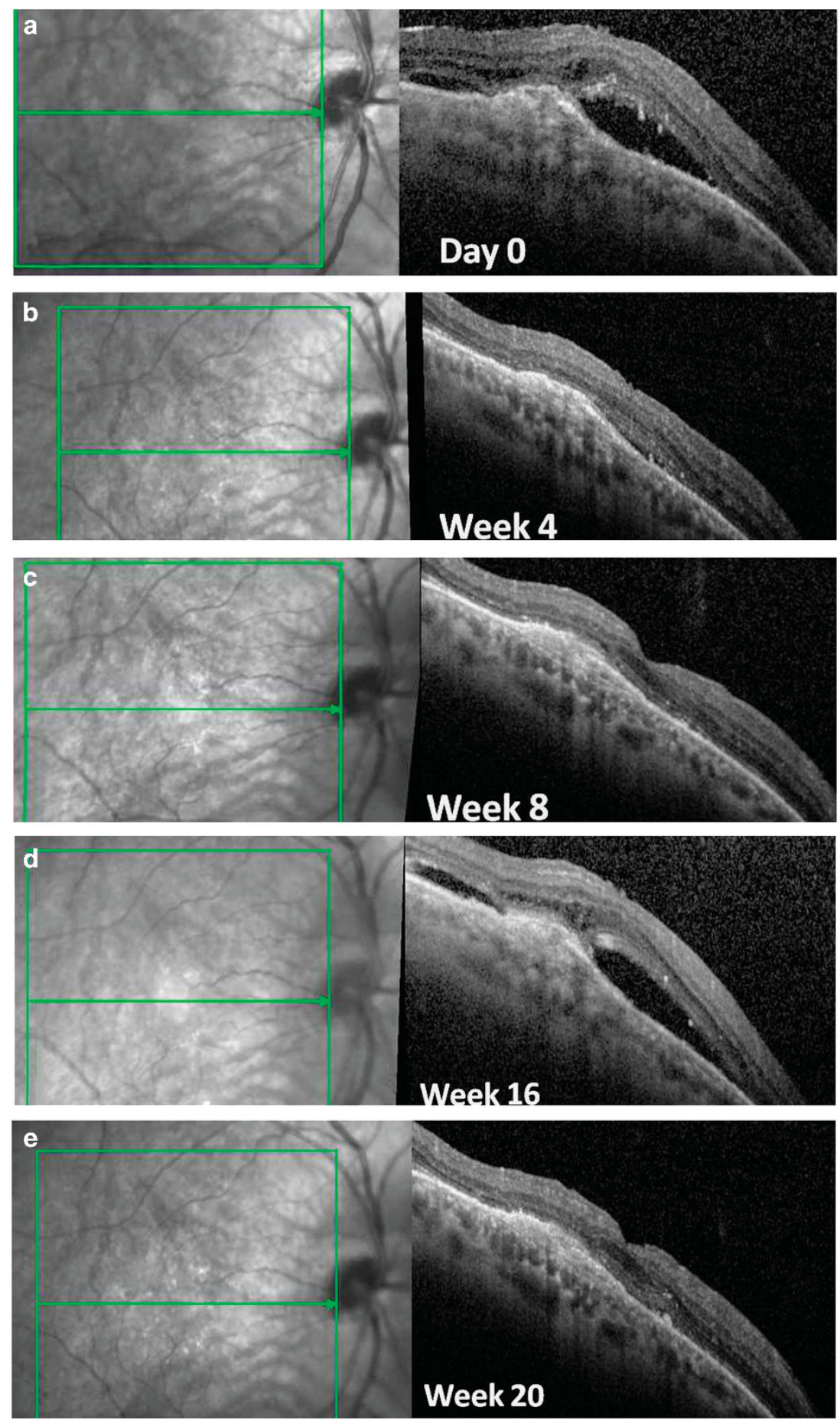

Figure 3 Seventy-four-year-old female who previously received five bevacizumab injections had SRF, IRF, and PED at baseline (a). Following the first aflibercept injection there was complete absorption of the IRF and minimal remnants of SRF (b), which resolved at week 8 (c). However, after the first bi-monthly interval, at week 16 there was recurrence of SRF and IRF (d). At week 20 there was a resolution of the fluid (e) and the macula remained dry until the end of the study under monthly therapy.

bevacizumab. Furthermore, we have evaluated patients at week 28, 4 weeks rather than 8 weeks after the last aflebercept injection, a factor that may have also favorably affected outcome. Yet, as mentioned above, previous prospective studies that enrolled eyes following longer period of prior first-line therapy also reported visual gain similar to the ASLI trial once conversion to aflibercept treatment was performed. Thus, it is likely that 
conversion can at least shorten the time interval for visual improvement in eyes with incomplete response to firstline therapy.

In conclusion, in the ASLI study, aflibercept yielded both anatomical and functional benefits following incomplete response to first-line bevacizumab therapy in nvAMD. Further studies should explore the benefit of second-line anti-VEGF therapies to provide data that facilitate the establishment of treatment protocols designed according to evidence-based data.

\section{Summary}

What was known before

- Aflibercept may improve both anatomical outcome and vision as a second-line treatment following incomplete response to ranibizumab in nvAMD.

\section{What this study adds}

- Aflibercept therapy improves anatomical and visual outcome in nvAMD eyes that had incomplete response to first-line bevacizumab therapy. Majority of eyes required monthly second-line aflibercept therapy during the first 6 months to preserve a fluid-free macula. Magnitude of anatomical and visual response may be similar regardless whether second-line therapy is commenced after few months of first-line therapy or later. Yet, final outcome may depend on the initial visual acuity.

\section{Conflict of interest}

Dr Chowers serves as consultant for Lycored, Biokine, Bayer, and Novartis; Dr Goldstein serves as a consultant and invited lecturer for Novartis, Allergan, and Bayer; and Dr Polack serves as a consulted for Allergan and Bayer. The remaining authors declare no conflict of interest.

\section{Acknowledgements}

The study was partially funded by Bayer. The study medication was provided by Bayer.

\section{Disclaimer}

The conception and conduct of the study as well as the analysis of the data were performed independently by the authors and the company had no control over the data.

\section{References}

1 Martin DF, Maguire MG, Ying GS, Grunwald JE, Fine SL, Jaffe GJ. Ranibizumab and bevacizumab for neovascular age-related macular degeneration. N Engl J Med 2011; 364: 1897-1908.
2 Chakravarthy U, Harding SP, Rogers CA, Downes SM, Lotery AJ, Wordsworth $\mathrm{S}$ et al. Ranibizumab versus bevacizumab to treat neovascular age-related macular degeneration: one-year findings from the IVAN randomized trial. Ophthalmology 2012; 119: 1399-1411.

3 Kodjikian L, Souied EH, Mimoun G, Mauget-Faysse M, Behar-Cohen F, Decullier E et al. Ranibizumab versus bevacizumab for neovascular age-related macular degeneration: results from the GEFAL noninferiority randomized trial. Ophthalmology 2013; 120: 2300-2309.

4 Krebs I, Schmetterer L, Boltz A, Told R, Vecsei-Marlovits V, Egger $\mathrm{S}$ et al. A randomised double-masked trial comparing the visual outcome after treatment with ranibizumab or bevacizumab in patients with neovascular age-related macular degeneration. Br J Ophthalmol 2013; 97: 266-271.

5 Berg K, Pedersen TR, Sandvik L, Bragadottir R. Comparison of ranibizumab and bevacizumab for neovascular agerelated macular degeneration according to LUCAS treatand-extend protocol. Ophthalmology 2015; 122: 146-152.

6 Heier JS, Brown DM, Chong V, Korobelnik JF, Kaiser PK, Nguyen QD et al. Intravitreal aflibercept (VEGF trap-eye) in wet age-related macular degeneration. Ophthalmology 2012; 119: 2537-2548.

7 Papadopoulos N, Martin J, Ruan Q, Rafique A, Rosconi MP, Shi E et al. Binding and neutralization of vascular endothelial growth factor (VEGF) and related ligands by VEGF Trap, ranibizumab and bevacizumab. Angiogenesis 2012; 15: 171-185.

8 Singh RP, Srivastava SK, Ehlers JP, Silva FQ, Bedi R, Schachat AP et al. A single-arm, investigator-initiated study of the efficacy, safety, and tolerability of intravitreal aflibercept injection in subjects with exudative age-related macular degeneration previously treated with ranibizumab or bevacizumab (ASSESS study): 12-month analysis. Clin Ophthalmol 2015; 9: 1759-1766.

9 Dvorak HF. Vascular permeability factor/vascular endothelial growth factor: a critical cytokine in tumor angiogenesis and a potential target for diagnosis and therapy. J Clin Oncol 2002; 20: 4368-4380.

10 Rakic JM, Lambert V, Devy L, Luttun A, Carmeliet P, Claes C et al. Placental growth factor, a member of the VEGF family, contributes to the development of choroidal neovascularization. Invest Ophthalmol Vis Sci 2003; 44: 3186-3193.

11 Ogura Y, Terasaki H, Gomi F, Yuzawa M, Iida T, Honda M et al. Efficacy and safety of intravitreal aflibercept injection in wet age-related macular degeneration: outcomes in the Japanese subgroup of the VIEW 2 study. Br J Ophthalmol 2015; 99: 92-97.

12 Ehlken C, Jungmann S, Bohringer D, Agostini HT, Junker B, Pielen A. Switch of anti-VEGF agents is an option for nonresponders in the treatment of AMD. Eye 2014; 28: 538-545.

13 Stepien KE, Rosenfeld PJ, Puliafito CA, Feuer W, Shi W, Al-Attar L et al. Comparison of intravitreal bevacizumab followed by ranibizumab for the treatment of neovascular age-related macular degeneration. Retina 2009; 29: 1067-1073.

14 Singh RP, Srivastava S, Ehlers JP, Bedi R, Schachat AP, Kaiser PK. A single-arm, investigator-initiated study of the efficacy, safety and tolerability of intravitreal aflibercept injection in subjects with exudative age-related macular degeneration, previously treated with ranibizumab or 
bevacizumab: 6-month interim analysis. $\mathrm{Br}$ J Ophthalmol 2014; 98: i22-i27.

15 Arcinue CA, Ma F, Barteselli G, Sharpsten L, Gomez ML, Freeman WR. One-year outcomes of aflibercept in recurrent or persistent neovascular age-related macular degeneration. Am J Ophthalmol 2015; 159: 426-436.

16 Bakall B, Folk JC, Boldt HC, Sohn EH, Stone EM, Russell SR et al. Aflibercept therapy for exudative age-related macular degeneration resistant to bevacizumab and ranibizumab. $A m$ J Ophthalmol 2013; 156: 15-22.

17 Chang AA, Li H, Broadhead GK, Hong T, Schlub TE, Wijeyakumar W et al. Intravitreal aflibercept for treatmentresistant neovascular age-related macular degeneration. Ophthalmology 2014; 121: 188-192.

18 Cho H, Shah CP, Weber M, Heier JS. Aflibercept for exudative AMD with persistent fluid on ranibizumab and/or bevacizumab. Br J Ophthalmol 2013; 97: 1032-1035.

19 Fassnacht-Riederle H, Becker M, Graf N, Michels S. Effect of aflibercept in insufficient responders to prior anti-VEGF therapy in neovascular AMD. Graefes Arch Clin Exp Ophthalmol 2014; 252: 1705-1709.

20 Gharbiya M, Iannetti L, Parisi F, De Vico U, Mungo ML, Marenco M. Visual and anatomical outcomes of intravitreal aflibercept for treatment-resistant neovascular age-related macular degeneration. BioMed Res Int 2014; 2014: 273754.

21 Grewal DS, Gill MK, Sarezky D, Lyon AT, Mirza RG. Visual and anatomical outcomes following intravitreal aflibercept in eyes with recalcitrant neovascular age-related macular degeneration: 12-month results. Eye 2014; 28: 895-899.

22 Griffin DR, Richmond PP, Olson JC. Intravitreal aflibercept outcomes in patients with persistent macular exudate previously treated with bevacizumab and/or ranibizumab for neovascular age-related macular degeneration. J Ophthalmol 2014; 2014: 497178.

23 Hall LB, Zebardast N, Huang JJ, Adelman RA. Aflibercept in the treatment of neovascular age-related macular degeneration in previously treated patients. J Ocul Pharmacol Ther 2014; 30: 346-352.

24 Heussen FM, Shao Q, Ouyang Y, Joussen AM, Muller B. Clinical outcomes after switching treatment from intravitreal ranibizumab to aflibercept in neovascular age-related macular degeneration. Graefe's Arch Clin Exp Ophthalmol 2014; 252: 909-915.

25 Ho VY, Yeh S, Olsen TW, Bergstrom CS, Yan J, Cribbs BE et al. Short-term outcomes of aflibercept for neovascular age-related macular degeneration in eyes previously treated with other vascular endothelial growth factor inhibitors. Am J Ophthalmol 2013; 156: 23-28.

26 Kawashima Y, Oishi A, Tsujikawa A, Yamashiro K, Miyake M, Ueda-Arakawa $\mathrm{N}$ et al. Effects of aflibercept for ranibizumabresistant neovascular age-related macular degeneration and polypoidal choroidal vasculopathy. Graefe's Arch Clin Exp Ophthalmol 2015; 253: 1471-1477.

27 Kumar N, Marsiglia M, Mrejen S, Fung AT, Slakter J, Sorenson $\mathrm{J}$ et al. Visual and anatomical outcomes of intravitreal aflibercept in eyes with persistent subfoveal fluid despite previous treatments with ranibizumab in patients with neovascular age-related macular degeneration. Retina 2013; 33: 1605-1612.

28 Messenger WB, Campbell JP, Faridi A, Shippey L, Bailey ST, Lauer AK et al. Injection frequency and anatomic outcomes
1 year following conversion to aflibercept in patients with neovascular age-related macular degeneration. Br J Ophthalmol 2014; 98: 1205-1207.

29 Patel KH, Chow CC, Rathod R, Mieler WF, Lim JI, Ulanski LJ 2nd et al. Rapid response of retinal pigment epithelial detachments to intravitreal aflibercept in neovascular agerelated macular degeneration refractory to bevacizumab and ranibizumab. Eye 2013; 27: 663-667.

30 Sarao V, Parravano M, Veritti D, Arias L, Varano M, Lanzetta P. Intravitreal aflibercept for choroidal neovascularization due to age-related macular degeneration unresponsive to ranibizumab therapy. Retina 2016; 36: 770-777.

31 Thorell MR, Nunes RP, Chen GW, Doshi RR, Dugar J, George MK et al. Response to aflibercept after frequent re-treatment with bevacizumab or ranibizumab in eyes with neovascular AMD. Ophthalmic Surg Lasers Imaging Retina 2014; 45: 526-533.

32 Wykoff CC, Brown DM, Maldonado ME, Croft DE. Aflibercept treatment for patients with exudative age-related macular degeneration who were incomplete responders to multiple ranibizumab injections (TURF trial). Br J Ophthalmol 2014; 98: 951-955.

33 Yonekawa Y, Andreoli C, Miller JB, Loewenstein JI, Sobrin L, Eliott D et al. Conversion to aflibercept for chronic refractory or recurrent neovascular age-related macular degeneration. Am J Ophthalmol 2013; 156: 29-35.

34 El-Mollayess GM, Mahfoud Z, Schakal AR, Salti HI, Jaafar D, Bashshur ZF. Fixed-interval versus OCT-guided variable dosing of intravitreal bevacizumab in the management of neovascular age-related macular degeneration: a 12-month randomized prospective study. Am J Ophthalmol 2012; 153: 481-489.

35 Tufail A, Patel PJ, Egan C, Hykin P, da Cruz L, Gregor Z et al. Bevacizumab for neovascular age related macular degeneration (ABC Trial): multicentre randomised double masked study. BMJ 2010; 340: c2459.

36 Homer N, Grewal DS, Mirza RG, Lyon AT, Gill MK. Transitioning to intravitreal aflibercept following a previous treat-and-extend dosing regimen in neovascular age-related macular degeneration: 24-month results. Eye 2015; 29: 1152-1155.

37 Mahmood S, Roberts SA, Aslam TM, Parkes J, Barugh KBishop PN, et al. Routine versus as-needed bevacizumab with 12-weekly assessment intervals for neovascular age-related macular degeneration: 92-week results of the GMAN Trial . Ophthalmology 2015; 122: 1348-1355.

38 Beykin G, Grunin M, Averbukh E, Banin E, Hemo Y, Chowers I. Bevacizumab treatment for neovascular agerelated macular degeneration in the setting of a clinic: 'real life' long-term outcome. BMC Ophthalmol 2015; 15: 39.

39 Grover S, Murthy RK, Brar VS, Chalam KV. Comparison of retinal thickness in normal eyes using Stratus and Spectralis optical coherence tomography. Invest Ophthalmol Vis Sci 2010; 51: 2644-2647.

40 Lazzeri S, Ripandelli G, Sartini MS, Parravano M, Varano M, Nardi $\mathrm{M}$ et al. Aflibercept administration in neovascular agerelated macular degeneration refractory to previous antivascular endothelial growth factor drugs: a critical review and new possible approaches to move forward. Angiogenesis 2015; 18: 397-432. 